\title{
Fractal Tie Dyeing Pattern Design based on Visual Basic Language Ning Tang ${ }^{1}$, Jianxia Su ${ }^{1, a}$ and Hongyi Chen ${ }^{1}$ \\ ${ }^{1}$ Information school of Beijing Institute Of Fashion Technology 614box ,100029 China \\ a gxysujx@bift.edu.cn
}

Key words: Fractal algorithm, Pattern, VB language

Abstract. In this paper, VB language is used to realize the design of fractal tie dyeing pattern, and apply Iterative and recursive algorithm to the design of fractal pattern. Koch curve algorithm is used to realize the design of fractal tree recursion and iteration function of affine transformation to realize iterative function pattern of graphic design, The simulation results show that the fractal algorithm can be applied to tie dyeing patterns and other broader areas.

\section{Introduction}

Fractal can be regarded as a mathematical tool to describe nature. Many objects in nature have "self similarity" and "self affine", and its unique fine structure. One of the core features of fractal is the fine structure, which can be seen in many times and the structure of the whole structure. Fractal has a great effect in many fields, for example, in nature, according to the field of view to observe the change and migration of address, whether it is a coastline or urban construction, has the characteristics of fractal.

Traditional labor people love a variety of patterns to decorate the daily life of accessories, dress is no exception. Extensive source of dye patterns, rich themes, including plants and flowers, birds and fish, geometric patterns and so on, The characteristics of tie dyeing technology determines the patterns in the form of common a single pattern, sequential patterns, symmetric patterns and integrative pattern. Today a hot topic is using computer software to simulate the traditional tie dyeing pattern.

\section{The design of fractal tie dyeing patterns}

Fractal patterns in the modern social life show the charm of its own, more and more is applied in all walks of life.

\section{Iterative algorithm for fractal pattern design}

\section{Design of fractal pattern based on iterative function system}

Iterated function system

While... Wend statement,

While condition.

$<$ statement block $>$

Wend

First, define the required variables, and then adjust the IFS code group according to the pattern, which needs a large number of experiments, to adjust the required style.

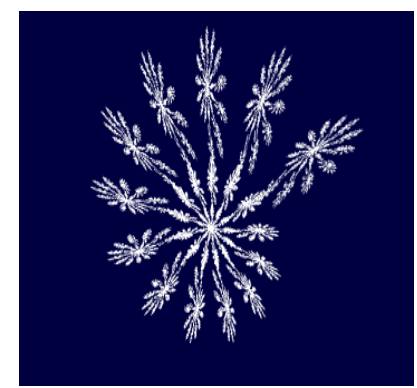

Fig. 1 Symmetrical fractal 
Fig. 1 is similar to the pattern of the chrysanthemum, but not the symmetrical pattern, if used for decoration, more active and more novel than the symmetrical pattern. For the whole program page, a single flower and can not hold up the table, so the next step of research purposes let a flower change into the flowers. the programming is used to assign iterations to distinguish various flowers variable repeat calls, using repeated calls to the cycle to complete the interface look like roses, or chrysanthemum, as shown in Fig. 2.

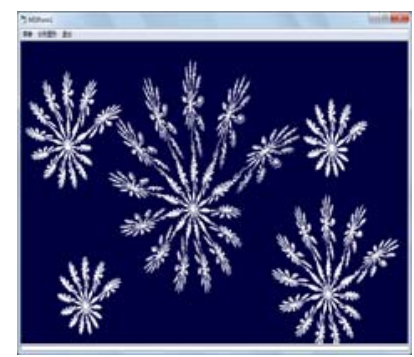

Fig. 2 Simulation of chrysanthemum

The important feature of fractal pattern is self-similarity, so if it can be designed to design a local and the overall pattern of similar patterns that would be better. Therefore based on this idea, after programming, the pattern appears similar to the style, as shown in Fig.3.

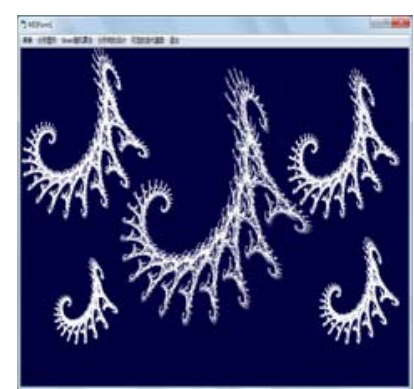

Fig.3 China Dragon diagram

At the same time, the model of the IFS is used to add the interface, and the interface shows rich.

\section{Julia function in the complex plane.}

Complex is a concept of mathematics. It contains the real and imaginary parts, such as $\mathrm{z}=\mathrm{a}+\mathrm{bi}$, a for the real part of complex, B is the imaginary part of the complex, where the design for the $\mathrm{X}$ axis, the $\mathrm{B}$ are arranged for the $\mathrm{Y}$ axis, it can draw a plane, called the complex plane.

The two function expression of the Julia collection is $\mathrm{F}(\mathrm{Z})=\mathrm{Z2}+\mathrm{C}(\mathrm{Z} 2$ and $\mathrm{C})$.

Set $\mathrm{Z} 0$ as a point, make $F(Z)=Z 2+C=Z 0$, the value of the two inverse function.

$\omega_{1}(Z)=\sqrt{Z-C}$

$\omega_{2}(Z)=-\sqrt{Z-C}$

Will $\left(\omega_{1}, \omega_{2}, C\right)$ as a IFS combination, and then take the probability of $\mathrm{P} 1=\mathrm{P} 2=1 / 2$, respectively, painting $\omega_{1}, \omega_{2}$. Its concrete steps are as follows:

$\mathrm{k}=0$ ( $\mathrm{k}$ is the number of iterations), the stack, draw point $\mathrm{Z} 0$

get a little $(Z, K)$ from the top of the stack.

according to the probability P1 and P2 were calculated Omega $\omega 1(\mathrm{z})=\sqrt{Z-C}$, Omega $\omega 2(\mathrm{z})$ $=-\sqrt{Z-C}$, painting point Omega $\omega$ 1and Omega $\omega 2$, Omega 2 , and $\mathrm{K}+1$ ) stack, make $\mathrm{z}=$ Omega $\omega 1$.

repeat step 3 until $\mathrm{k}=\mathrm{n}-1$

draw points $\sqrt{Z-C},-\sqrt{Z-C}$.

to determine whether the stack is empty, if the stack is empty, otherwise repeat step 2-6

The real and imaginary part of the $\mathrm{C}$ will also bring some impact to the program.

Draw a similar tie dye small Sui hua pictorial design here, so the use of navy blue color, white 
color style, when the initial value $\mathrm{C}=-0.46+0.57 \mathrm{i}$, the resulting pattern as shown in Fig. 4:

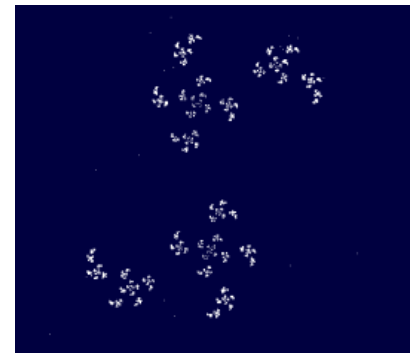

Fig.4 Julia complex plane iteration small Suihua renderings

By changing the $\mathrm{CX}$ and $\mathrm{CY}$ value pattern transformation point falls in the area, change if RND $<0.83333$ value to change the placement of probability.

To summarize and explore, on $\mathrm{C}$, the real part and the imaginary part of the debugging, found that there are still a lot of similar pattern, as shown in Figure 5 and Figure 6:

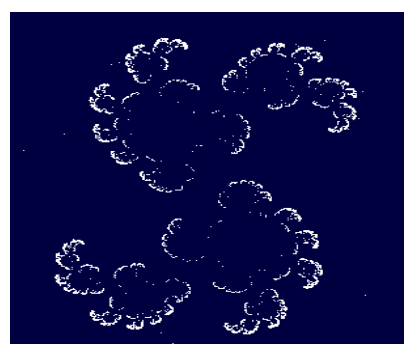

Fig.5 Julia complex plane iterative fireworks display

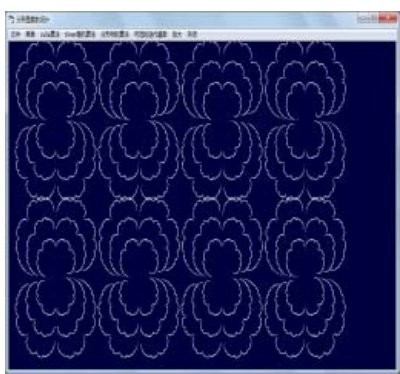

Fig.6 Julia complex plane iterative peony flower

Snowflake shape has a lot of style, so as to change the way of thinking, imagine snowflakes are nature is very peculiar shape, every flower snow is different style, so only a kind of flowers here is under study. In the symmetric pattern snow, research is still relatively easy. But it is innovation, so Asymmetry in the symmetric is selected the to program, see Figure 7:

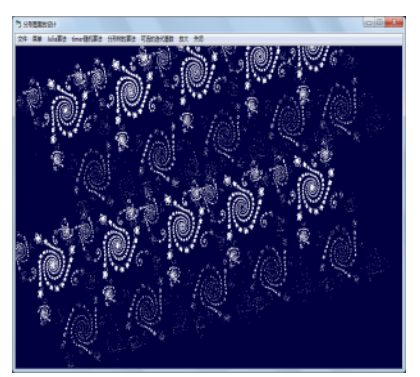

Fig.7 Julia complex plane iteration simulation of snowflake

\section{Conclusions}

The fractal theory provides a more accurate description of the complex scenes in nature. This paper programming is based on the theory of fractal theory. The fractal pattern is a combination of 
mathematical calculation and natural science or art design. In this paper, the of the computer realized the simulation of traditional tie dyeing pattern, most of fractal tie dyeing pattern design is not very complex but the result shows that it is a very effective way. Though it still needs to continue in-depth study. The study can go on better under more time and work.

\section{Acknowledgments}

This paper is sponsored by KYJH02140201/025 of 2014 project of Beijing Science and Technology.

\section{References}

[1] Shixian Qu, Jianhua Zhang. Fractal theory and application of complex system. Xi'an: Shanxi people's Press,. 1996.

[2] Ruian Hu, Jiyang Hu. Factal image and its application. China Railway Press, 1995

[3] Kenneth Falcone. One mathematical basis of fractal geometry and its application Northeast University Press.1991.

[4] Bowen Sun. Fractal algorithm and program design - C++ Visual to achieve [M]. Beijing: Science Press, 2004

[5] Shuigen Li.Fractal graph of two-dimensional and high dimensional space. Science Press, 2009 